\title{
SARS-CoV-2 and the Possible Role of Raf/MEK/ERK Pathway in Viral Survival: Is This a Potential Therapeutic Strategy for COVID-19?
}

\author{
Morteza Ghasemnejad-Berenji ${ }^{a}$ Sarvin Pashapour ${ }^{b}$ \\ aDepartment of Pharmacology and Toxicology, Faculty of Pharmacy, Urmia University of Medical Sciences, Urmia, Iran; \\ ${ }^{b}$ Department of Pediatrics, Faculty of Medicine, Motahari Hospital, Urmia University of Medical Sciences, Urmia, Iran
}

\section{Keywords \\ COVID-19 · Raf/MEK/ERK signaling $\cdot$ RNA viruses $\cdot$ Viral life cycle $\cdot$ Viral survival}

In late 2019, a sudden rise in respiratory-related disease cases in China triggered the identification of its source as a novel corona virus, termed severe acute respiratory syndrome coronavirus 2 (SARS-CoV-2). The disease caused by novel SARS-CoV-2 is named as COVID-19 by the World Health Organization [1]. The culprit virus belongs to the Coronaviridae family of coronaviruses that caused 2 other outbreaks, namely, severe acute respiratory syndrome (SARS) in 2002 [2] and Middle East respiratory syndrome in 2012 [3]. As the survival of every virus depends on its host cell, the understanding of cellular functions such as signaling pathways that are essential for viral replication may be suitable to define targets for antiviral therapy and pave the way toward effective drugs against essential cellular activities supporting viral replication [4]. In this regard, we have focused on the Raf/ MEK/ERK signaling pathway, which is probably one of the most well-known signal transduction pathways among biologists because of its implication in a wide variety of cellular functions such as cell proliferation, cell cycle arrest, and apoptosis [5]. The mechanism of this pathway is initiated by $G$ protein-coupled receptors, which leads to the phosphorylation of downstream molecules and activates the serine threonine kinase Raf (dual specificity kinase MEK and MAPK/ERK). ERK phosphorylates various substrates, transforms the signals, and follows different functions in cells [6]. Hence, it is not surprising that several DNA and RNA viruses inherit this pathway, apart from an initial activation upon viral attachment, for various steps in the viral life cycle [7]. Consequently, the kinetic of pathway activation is highly dynamic [8]. For example, herpes simplex type-1 virus-induced activation of the Raf/MEK/ERK pathway is used for cytoskeleton rearrangement during entry [9], JC polyomavirus requires ERK activation for viral transcription [10], and influenza A virus hijacks Raf/MEK/ERK activity for efficient viral ribonucleoprotein export [11, 12]. Ebola virus glycoprotein-induced cytotoxicity depends on ERK activation [13], and hepatitis $C$ virus relies on Raf/MEK/ERK-mediated upregulation of cytosolic phospholipase A2 for efficient particle production [14]. Flaviviruses, including yellow fever virus, Saint-Louis encephalitis virus, and dengue virus, as well depend on Raf/ MEK/ERK-mediated signaling for efficient replication karger@karger.com

(C) 2020 S. Karger AG, Basel

www.karger.com/pha

Karger"
Morteza Ghasemnejad-Berenji

Department of Pharmacology and Toxicology, Faculty of Pharmacy

Urmia University of Medical Sciences

Nazlou, Urmia (Iran)

ghasemnejad.m@umsu.ac.ir 
$[15,16]$. It has been shown that activated ERK1/2 enhanced the infectivity of human immunodeficiency virus, whereas treatment of cells with the MEK1/2 inhibitor PD98059 significantly inhibited human immunodeficiency virus infectivity $[17,18]$. Treating cells with UO126, a highly selective inhibitor of both MEK1 and MEK2, also significantly inhibited the propagation of influenza A virus, Borna disease virus, coxsackievirus B3, and HCMV [11, 19-21]. Thus, it appears that the MEK1/2 inhibitors have a broad effect on propagations of viruses from various families (positive-strand and negativestrand RNA viruses, retroviruses, and DNA viruses) [22]. SARS-CoV-2 is an enveloped, positive-sense, singlestranded RNA beta-coronavirus. Similar to SARS and Middle East respiratory syndrome, the SARS-CoV-2 genome encodes nonstructural proteins (such as 3-chymotrypsin-like protease, papain-like protease, helicase, and RNA-dependent RNA polymerase), structural proteins (such as spike glycoprotein), and accessory proteins. The 4 nonstructural proteins mentioned above are key enzymes in the viral life cycle, and the spike glycoprotein is indispensable for virus-cell receptor interactions during viral entry [23]. By analyzing the effects of transiently expressed viral spike protein (S) of SARS-CoV, it was revealed that the $S$ protein plays an important role in virusstimulated cyclooxygenase-2 (COX-2) expression [24]. COX-2 is a prostaglandin synthetase involved in inflammation [25] that is highly regulated by different factors including cytokines [26]. The upstream calcium-dependent PKCa that modulates the downstream Raf/MEK/ ERK pathway is induced by the SARS-CoV S protein. It was revealed that ERK is involved in S protein-induced activation of the COX-2 promoter and the production of COX-2 protein in HEK293T cells. This result helps explain the function of SARS-CoV S protein in SARS pathogenesis [24]. More information on relevant MAPK signaling induced by coronavirus was obtained for murine coronavirus. It was revealed that infection of cultured cells with murine coronavirus resulted in activation of the Raf/MEK/ERK signal cascade, and inhibition of the MAPK signaling pathway by U0126 or knockdown of MEK and ERK by small interfering RNAs significantly impaired murine coronavirus progeny production. The treatment did not affect virus entry or cellular and viral mRNA production. However, synthesis of viral genomic and sub-genomic RNAs was severely suppressed by U0126 treatment. This study indicated that the MAPK signaling pathway is involved in murine coronavirus RNA synthesis [22]. Selective RAF inhibitors are well tolerated, and severe toxicities occur infrequently in noninfected cells. Among the common grade 1-2 adverse events are dermatological affections (50-70\%), fatigue (30$50 \%)$, diarrhea (10-30\%), and nausea (10-20\%) [27]. Considering the role of the Raf/MEK/ERK signaling pathway in the pathogenesis of various viruses, it is probable that the activation of this signaling pathway by $\mathrm{CO}$ VID-19 has an important role in the survival of this virus. In this regard, drugs that inhibit the Raf/MEK/ERK signaling pathway may be potential antiviral candidates for the treatment of COVID-19.

\section{Conflict of Interest Statement}

The authors have no conflicts of interest to declare.

\section{Funding Sources}

The authors did not receive any funding.

\section{Author Contributions}

M. Ghasemnejad-Berenji: literature review and writing the manuscript. S. Pashapour: literature review and revising the manuscript.

\section{References}

$1 \mathrm{Xu} \mathrm{Z}$, Shi L, Wang Y, Zhang J, Huang L, Zhang C, et al. Pathological findings of COVID-19 associated with acute respiratory distress syndrome. Lancet Respir Med. 2020; $8(4): 420-2$

2 Ruan YJ, Wei CL, Ee AL, Vega VB, Thoreau $\mathrm{H}$, Su ST, et al. Comparative full-length genome sequence analysis of 14 SARS coronavirus isolates and common mutations associated with putative origins of infection. Lancet. 2003;361(9371):1779-85.
3 Group WM-CR. State of knowledge and data gaps of Middle East respiratory syndrome coronavirus (MERS-CoV) in humans. PLoS Curr. 2013;5.

4 Ludwig S, Planz O. Influenza viruses and the NF-kappaB signaling pathway: towards a novel concept of antiviral therapy. Biol Chem. 2008;389(10):1307-12.

5 Peyssonnaux C, Eychène $\mathrm{A}$. The Raf/MEK/ ERK pathway: new concepts of activation. Biol Cell. 2001;93(1-2):53-62.
6 Li L, Zhao GD, Shi Z, Qi LL, Zhou LY, Fu ZX. The Ras/Raf/MEK/ERK signaling pathway and its role in the occurrence and development of HCC. Oncol Lett. 2016;12(5):3045-50.

7 Preugschas HF, Hrincius ER, Mewis C, Tran GVQ, Ludwig S, Ehrhardt C. Late activation of the Raf/MEK/ERK pathway is required for translocation of the respiratory syncytial virus $\mathrm{F}$ protein to the plasma membrane and efficient viral replication. Cell Microbiol. 2019; 21(1):e12955. 
8 Bonjardim CA. Viral exploitation of the MEK/ERK pathway: a tale of vaccinia virus and other viruses. Virology. 2017;507:267-75.

9 Qin D, Feng N, Fan W, Ma X, Yan Q, Lv Z, et al. Activation of PI3K/AKT and ERK MAPK signal pathways is required for the induction of lytic cycle replication of Kaposi's sarcomaassociated herpesvirus by herpes simplex virus type 1. BMC Microbiol. 2011;11(1):240.

10 DuShane JK, Wilczek MP, Mayberry CL, Maginnis MS. ERK is a critical regulator of JC polyomavirus infection. J Virol. 2018;92(7): e01529-17.

11 Pleschka S, Wolff T, Ehrhardt C, Hobom G, Planz O, Rapp UR, et al. Influenza virus propagation is impaired by inhibition of the Raf/ MEK/ERK signalling cascade. Nat Cell Biol. 2001;3(3):301-5.

12 Pinto R, Herold S, Cakarova L, Hoegner K, Lohmeyer J, Planz O, et al. Inhibition of influenza virus-induced NF-kappaB and Raf/ MEK/ERK activation can reduce both virus titers and cytokine expression simultaneously in vitro and in vivo. Antiviral Res. 2011;92(1): $45-56$.

13 Zampieri CA, Fortin JF, Nolan GP, Nabel GJ. The ERK mitogen-activated protein kinase pathway contributes to Ebola virus glycoprotein-induced cytotoxicity. J Virol. 2007;81(3): 1230-40.

14 Zhang Q, Gong R, Qu J, Zhou Y, Liu W, Chen $M$, et al. Activation of the Ras/Raf/MEK pathway facilitates hepatitis $C$ virus replication via attenuation of the interferon-JAK-STAT pathway. J Virol. 2012;86(3):1544-54.
15 Shyu HW, Lin YY, Chen LC, Wang YF, Yeh $\mathrm{TM}, \mathrm{Su}$ SJ, et al. The dengue virus envelope protein induced PAI-1 gene expression via MEK/ERK pathways. Thromb Haemost. 2010;104(6):1219-27.

16 Albarnaz JD, De Oliveira LC, Torres AA, Palhares RM, Casteluber MC, Rodrigues CM, et al. MEK/ERK activation plays a decisive role in yellow fever virus replication: implication as an antiviral therapeutic target. Antiviral Res. 2014;111:82-92.

17 Rusnati M, Urbinati C, Musulin B, Ribatti D, Albini A, Noonan D, et al. Activation of endothelial cell mitogen activated protein kinase ERK(1/2) by extracellular HIV-1 Tat protein. Endothelium. 2001;8(1):65-74.

18 Gong J, Shen XH, Chen C, Qiu H, Yang RG. Down-regulation of HIV-1 infection by inhibition of the MAPK signaling pathway. Virol Sin. 2011;26(2):114-22.

19 Johnson RA, Ma XL, Yurochko AD, Huang ES. The role of MKK1/2 kinase activity in human cytomegalovirus infection. J Gen Virol. 2001;82(Pt 3):493-7.

20 Planz O, Pleschka S, Ludwig S. MEK-specific inhibitor U0126 blocks spread of Borna disease virus in cultured cells. J Virol. 2001; 75(10):4871-7.
21 Luo H, Yanagawa B, Zhang J, Luo Z, Zhang M, Esfandiarei M, et al. Coxsackievirus B3 replication is reduced by inhibition of the extracellular signal-regulated kinase (ERK) signaling pathway. J Virol. 2002;76(7):3365-73.

22 Cai Y, Liu Y, Zhang X. Suppression of coronavirus replication by inhibition of the MEK signaling pathway. J Virol. 2007;81(2):44656.

23 ul Qamar MT, Alqahtani SM, Alamri MA, Chen L-L. Structural basis of SARS-CoV-2 3CLpro and anti-COVID-19 drug discovery from medicinal plants. Journal of pharmaceutical analysis. 2020;10(4):313-9.

24 Liu M, Yang Y, Gu C, Yue Y, Wu KK, Wu J, et al. Spike protein of SARS-CoV stimulates cyclooxygenase- 2 expression via both calcium-dependent and calcium-independent protein kinase C pathways. FASEB J. 2007; 21(7):1586-96.

25 Ferrer MD, Busquets-Cortés C, Capó X, Tejada S, Tur JA, Pons A, et al. Cyclooxygenase-2 inhibitors as a therapeutic target in inflammatory diseases. Curr Med Chem. 2019; 26(18):3225-41.

26 Machado-Carvalho L, Roca-Ferrer J, Picado C. IL-4/IFN- $\gamma$ inflammatory cytokine profile induces a deficient regulation of the IL- $1 \beta / \mathrm{IL}$ 1RI/EP. Respir Med. 2019;150:136-40.

27 Manousaridis I, Mavridou S, Goerdt S, Leverkus M, Utikal J. Cutaneous side effects of inhibitors of the RAS/RAF/MEK/ERK signalling pathway and their management. J Eur Acad Dermatol Venereol. 2013;27(1):11-8. 